\title{
Prognostic value of combined pretreatment fibrinogen and neutrophil-lymphocyte ratio in digestive system cancers: a meta-analysis of 17 retrospective studies
}

\author{
Rongqiang Liu ${ }^{1,2 \#}$, Tianxing Dai ${ }^{1 \#}$, Shiyang Zheng ${ }^{3 \#}$, Mingbin Deng ${ }^{1}$, Guozhen Lin ${ }^{1}$, Yuanda Bao ${ }^{2}$, \\ Zhihua Guo ${ }^{4}$, Guoying Wang ${ }^{1}$ \\ ${ }^{1}$ Department of Hepatic Surgery and Liver Transplantation Center, the Third Affiliated Hospital of Sun Yat-sen University, Guangzhou, China; \\ ${ }^{2}$ Department of Hepatobiliary Surgery, the First Affiliated Hospital of Guangzhou Medical University, Guangzhou, China; ${ }^{3}$ Department of Breast \\ Surgery, the Third Affiliated Hospital of Guangzhou Medical University, Guangzhou, China; ${ }^{4}$ Department of Thoracic Oncology, the First Affiliated \\ Hospital of Guangzhou Medical University, Guangzhou, China \\ Contributions: (I) Conception and design: Z Guo, G Wang; (II) Administrative support: G Wang; (III) Provision of study materials or patients: R Liu, \\ T Dai, S Zheng, M Deng, G Lin, Y Bao; (IV) Collection and assembly of data: R Liu, T Dai, S Zheng; (V) Data analysis and interpretation: R Liu, T \\ Dai, S Zheng; (VI) Manuscript writing: All authors; (VII) Final approval of manuscript: All authors. \\ \#These authors contributed equally to this work. \\ Correspondence to: Guoying Wang. Department of Hepatic Surgery and Liver transplantation Center, The Third Affiliated Hospital of Sun Yat-sen \\ University, No. 600, Tianhe Street, Tianhe District, Guangzhou 510220, China. Email: wanggy3@126.com; Zhihua Guo. Department of Thoracic \\ Oncology, the First Affiliated Hospital of Guangzhou Medical University, No. 151, Yanjiang West Road, Yuexiu District, Guangzhou, China. \\ Email: guozhihua84@126.com.
}

Background: Several epidemiological studies have reported the relationship between the combined pretreatment fibrinogen and neutrophil-lymphocyte ratio (F-NLR) and prognosis of digestive system cancers (DSCs). However, the results are controversial. We aimed to assess the prognostic value of F-NLR in patients with DSCs.

Methods: A comprehensive search for relevant studies was conducted until June, 2020. Studies that evaluated the association of the F-NLR score with survival outcome in patients with any DSCs were included. The hazard ratio (HR) and $95 \%$ confidence interval (CI) were calculated using a fixed-effects model. All data analyses were performed using the STATA 12.0 software.

Results: A total of 17 studies involving 5,767 participants were included in the meta-analysis. We found that high F-NLR score was significantly associated with poor overall survival (OS) in patients with DSCs ( $\mathrm{HR}=2.0$; 95\% CI, 1.78-2.24). In addition, patients with high F-NLR score had poor disease-free survival/ progression-free survival/recurrence-free survival (DFS/PFS/RFS) (HR =2.01; 95\% CI, 1.47-2.74) and DFS (HR $=1.97 ; 95 \%$ CI, 1.35-2.87). Sensitivity analyses for OS confirmed that the results were stable.

Conclusions: High F-NLR score is significantly associated with poor prognostic outcomes in patients with DSCs and can serve as an effective prognostic indicator for the Asian population.

Keywords: Fibrinogen; neutrophil-lymphocyte ratio; prognosis; digestive system cancers (DSCs); meta-analysis

Submitted Jul 06, 2020. Accepted for publication Nov 06, 2020.

doi: $10.21037 /$ tcr-20-2482

View this article at: http://dx.doi.org/10.21037/tcr-20-2482

\section{Introduction}

Digestive system cancers (DSCs) are the most common malignancies, composing approximately $30 \%$ of all cancers.
In the United States, nearly 350,000 new diagnosed DSC cases occur annually and approximately half die (1). Over 3.4 million new cases of DSCs and 1.5 million 
deaths are estimated to happen each year worldwide (2). The occurrence and development of DSCs are closely related to heredity, chronic disease history, lifestyle, and environmental factors. In recent years, the incidence of DSCs in developing countries has significantly increased. In China, stomach, esophageal, and liver cancers were also commonly diagnosed and were identified as leading causes of cancer death (3). Despite recent improvements in various detection and therapy methods, the prognosis of patients with DSCs remains unsatisfactory. Many different prognostic markers have been used for digestive system tumors, but the clinical application effect is not obvious. Therefore, it is essential to identify new more effective prognostic biomarkers for DSCs.

Recently, a new scoring system, termed fibrinogen and neutrophil-lymphocyte ratio (F-NLR), that combines pretreatment fibrinogen levels with neutrophil-lymphocyte ratio (NLR) has gradually attracted considerable research attention. The F-NLR score has been reported as a promising prognostic marker in patients with DSCs (4-20). However, the results remain controversial. Therefore, this study aimed to comprehensively determine the prognostic value of F-NLR score in patients with DSCs by integrating data in a meta-analysis. We present the following article in accordance with the PRISMA reporting checklist (available at http://dx.doi.org/10.21037/tcr-20-2482).

\section{Methods}

\section{Search strategy}

Three independent investigators (RL, TD and SZ) conducted a literature search. Relevant studies were systematically searched in PubMed, EMBASE, Web of Science, China National Knowledge Infrastructure, and Wanfang Data until June, 2020. The following key words were used: "fibrinogen" AND "neutrophil lymphocyte ratio" OR "neutrophil-lymphocyte ratio" OR "neutrophil to lymphocyte ratio" OR "neutrophil-lymphocyte-ratio" OR "neutrophil-lymphocyte "OR "NLR" AND "cancer" OR "carcinoma" OR "neoplasm" OR "tumour" OR "tumor" AND "prognosis" OR "prognostic" OR "survival” OR "outcome". Titles, abstracts, full texts, and reference lists were carefully screened to identify objective studies. There were no language restrictions and a manual search was conducted for references in the included studies.

\section{Study selection}

All articles were independently assessed by the three investigators (RL, TD and SZ). Discrepancies were resolved by consensus. The inclusion criteria for studies were: (I) evaluated the association of the F-NLR score with survival outcome in patients with any DSCs; and (II) provided sufficient data to allow calculation of the hazard ratio (HR) with $95 \%$ confidence interval (CI). The exclusion criteria were: studies with insufficient data; animal experiments; letters; case reports; and abstracts.

\section{Data extraction and quality assessment}

Data were independently extracted by two researchers (RL and SZ). A standardized data collection form was used to extract the following information: first author name, publication year, country, study design, tumor type, sample size, overall survival (OS), disease-free survival/progressionfree survival/recurrence-free survival (DFS/PFS/RFS), as well as the HR and the corresponding $95 \%$ CI. For studies reporting the results of both univariate and multivariate analyses, those obtained from the latter were selected, as this approach considers confounding factors and is more accurate. The quality of each study was assessed using the Newcastle-Ottawa Quality Assessment Scale (NOS) (21).

\section{Statistical analysis}

The HR and corresponding 95\% CI were used to analyze pooled data. Statistical variables described in the studies were directly used in the present analysis. Otherwise, the data were extracted from graphical survival plots according to the methods described by Tierney (22). Data from the Kaplan-Meier survival curves were analyzed using the Engauge Digitizer version 4.1 software. Heterogeneity was assessed based on $\mathrm{I}^{2}$. For $\mathrm{I}^{2}<50 \%$ and $\geq 50 \%$, fixedeffects and random-effects models were used, respectively. Sensitivity analysis was conducted to test the stability of the results. Begg's and Egger's tests were used to evaluate publication bias. All data analyses were performed using the STATA 12.0 software (Stata Corp., College Station, TX, USA). $\mathrm{P}$ values $<0.05$ denoted statistically significant differences.

\section{Results}

\section{Search results}

Through a systematic literature search of the designated databases, a total of 353 articles were initially collected. After removing 171 duplicates, 182 articles remained. After 


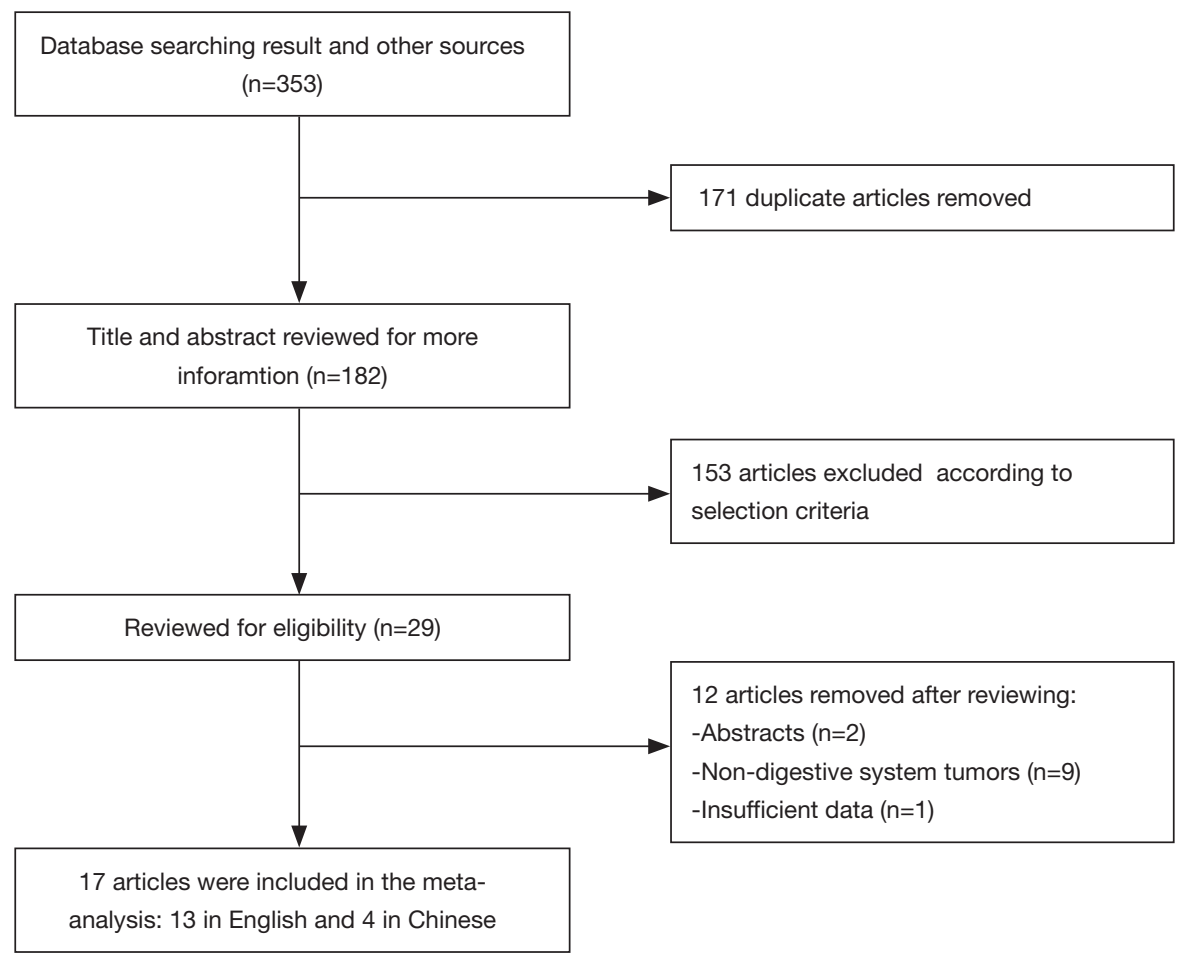

Figure 1 Flow diagram of the literature search.

screening the titles and abstracts, 153 articles which did not meet the inclusion criteria were removed. After fulltext review, 12 articles were further excluded. Eventually, 17 retrospective articles that investigated the association of the F-NLR score with the prognostic outcome in patients with DSCs were included in the final analysis. The flow chart for study identification is presented in Figure 1.

\section{Study characteristics}

The total number of patients in the included articles was 5,767 , ranging from 68 to 1,293 per study. Sixteen studies presented OS data, four reported DFS data, two covered PFS, and one reported RFS. Eleven studies were conducted in China, and six in Japan. Five different types of DSCs were assessed in this study, including esophageal carcinoma (EC) $(4,8,13,17,18)$, gastric cancer (GC) $(5,6,11,14,16,20)$, colorectal cancer $(\mathrm{CRC})(10,12,19)$, hypopharyngeal carcinoma (HPC) (9), hepatocellular carcinoma (HCC) $(7,15)$. The characteristics of the included studies are summarized in Table 1.

\section{F-NLR score on OS}

Sixteen studies, including 5,688 participants, focused on OS analysis. The fixed effects model was adopted since there was no heterogeneity $\left(\mathrm{I}^{2}=0 \%\right)$. The results of metaanalysis revealed that a high F-NLR score was significantly associated with poor OS in DSCs (HR: 2.0; $95 \%$ CI: 1.78-2.24) (Figure 2).

We performed subgroup analyses according to tumor type, country, analysis type and treatments (Table 2). The findings revealed that the high F-NLR score was an effective prognostic indicator for OS in GC (HR: 2.35; 95\% CI: 1.89-2.91), EC (HR: 1.82; 95\% CI: 1.50-2.22), HCC (HR: 2.12; 95\% CI: 1.52-2.94), and CRC (HR: 2.29; 95\% CI: 1.27-4.15). Regardless of gastrointestinal or non-gastrointestinal tract cancers, the high F-NLR score indicated poor OS (HR: 2.09; 95\% CI: $1.78-2.45$ and HR: 1.90; 95\% CI: 1.61-2.25, respectively). We also found that the high F-NLR score was obviously associated with unfavorable OS for the surgery group (HR: 1.90; 95\% CI: 1.67-2.17), no-surgery group (HR: $2.13 ; 95 \%$ CI: $1.47-$ 3.09), and mixed group (HR: 2.92; 95\% CI: 1.61-5.29). In 
Table 1 The basic information of included studies

\begin{tabular}{|c|c|c|c|c|c|c|c|c|c|}
\hline Study & Year & Country & Study type & Tumor type & Sample & $\begin{array}{l}\text { Treatment } \\
\text { methods }\end{array}$ & Analysis type & $\begin{array}{l}\text { Survival } \\
\text { analysis }\end{array}$ & NOS score \\
\hline Arigami & 2015 & Japan & Retrospective & ESCC & 238 & With-surgery & Multivariate- & os & 7 \\
\hline Arigami & 2016B & Japan & Retrospective & GC & 68 & With-surgery & Multivariate & os & 7 \\
\hline Kuwahara & 2018 & Japan & Retrospective & HPC & 111 & No-surgery & Univariate & OS, PFS & 8 \\
\hline $\mathrm{Li}$ & 2018 & China & Retrospective & CRC & 693 & With-surgery & Univariate & OS, DFS & 8 \\
\hline Liu & 2018 & China & Retrospective & GC & 1,293 & With-surgery & Multivariate & os & 7 \\
\hline Kong & 2020 & China & Retrospective & $\mathrm{HCC}$ & 292 & With-surgery & Multivariate & OS, DFS & 8 \\
\hline Yamamoto & 2020 & Japan & Retrospective & $\mathrm{GC}$ & 666 & Mixed & Univariate & OS, RFS & 8 \\
\hline Lin & 2019 & China & Retrospective & ESCC & 327 & With-surgery & Multivariate & os & 6 \\
\hline Feng & 2019 & China & Retrospective & ESCC & 218 & Mixed & Multivariate & os & 6 \\
\hline Qin & 2017 & China & Retrospective & CRC & 250 & With-surgery & Univariate & os & 6 \\
\hline
\end{tabular}

ESCC, esophageal squamous cell carcinoma; GC, gastric cancer; HCC, hepatocellular carcinoma; HPC, hypopharyngeal carcinoma; CRC, colorectal cancer; LARC, locally advanced rectal cancer; AEG, adenocarcinoma of the esophagogastric junction; UGC, upper gastric cancer; OS, overall survival; DFS, disease-free survival.

addition, in the subgroup based on country, the merged HRs were 2.22 (95\% CI: 1.71-2.88) and 1.95 (95\% CI: 1.71-2.21) for Japan and China, respectively.

\section{F-NLR score on DFS/PFS/RFS}

Seven studies involving 2,288 participants that reported DFS/ $\mathrm{PFS} / \mathrm{RFS}$ showed obvious heterogeneity $\left(\mathrm{I}^{2}=71.7 \%\right)$ (Figure 3 ). For these studies, we calculated the pooled HR using a random effects model. Comprehensive analysis indicated that the high F-NLR score was significantly associated with poor DFS/PFS/ RFS (HR: 2.01; 95\% CI: 1.47-2.74). Furthermore, data were analyzed based on DFS and RFS (Table 3). We found that the high F-NLR score might be a significant biomarker for DFS (HR =1.97; 95\% CI: 1.35-2.87), but was not associated with RFS (HR $=2.12$; 95\% CI: 0.65-6.88).

\section{Sensitivity analysis}

Sensitivity analysis was conducted by excluding each study in turn for OS and DFS/PFS/RFS. As shown in Figure 4, the results did not differ significantly from those of the overall analysis, revealing that the outcomes were stable.

\section{Publication bias}

The funnel plot was used to qualitatively determine the publication bias, and Egger's was employed to quantify the publication bias. As shown in Figures 5 and $6 \mathrm{P}$ values of Egger's for OS and DFS/PFS/RFS was 0.017 and 0.20, respectively, indicating there was publication bias for OS. Through the trim-and-fill method, we found that the pooled HR for OS was 1.776 (95\% CI: 1.609-1.961), further confirming that the result was unaffected.

\section{Discussion}

To the best of our knowledge, this is the first meta-analysis to comprehensively assess the prognostic value of the F-NLR score in DSCs. A total of 17 studies involving 5,767 


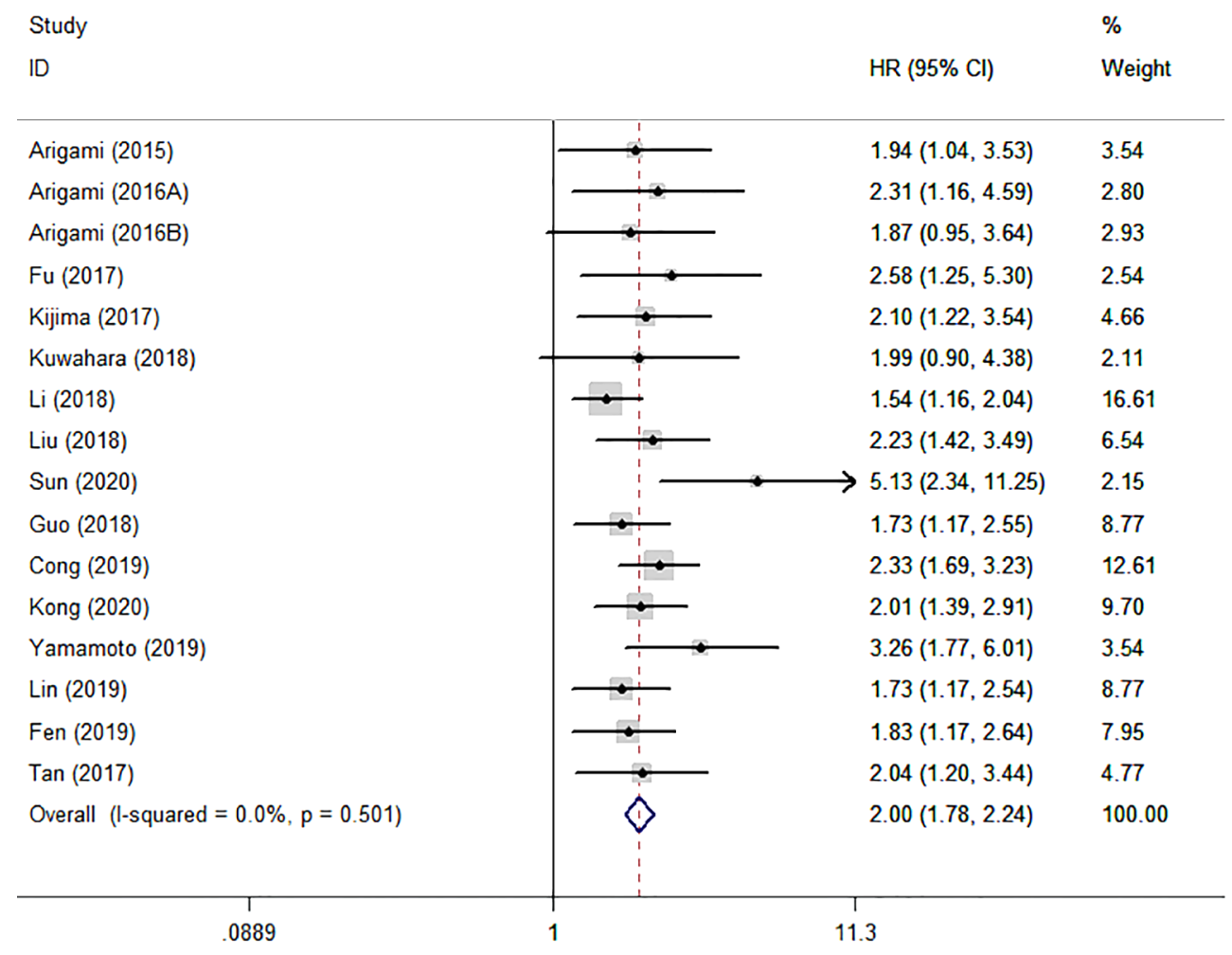

Figure 2 Forest plot of the relationship between pretreatment fibrinogen and neutrophil-lymphocyte ratio and overall survival.

patients were included. Of those, sixteen studies assessed the prognostic role of F-NLR score in OS and seven studies evaluated the prognostic role of the F-NLR score in DFS/ PFS/RFS. Our results demonstrated that the high F-NLR score was significantly associated with poor OS (HR: 2.0; 95\% CI: 1.78-2.24). Subgroup analysis for OS showed that the high F-NLR score mainly displayed the adverse prognosis in GC (HR: 2.35; 95\% CI: 1.89-2.91), ESCC (HR: 1.82; 95\% CI: 1.50-2.22), HCC (HR: 2.12; 95\% CI: 1.52-2.94), and CRC (HR: 2.29; 95\% CI: 1.27-4.15). In addition, the meta-analysis revealed that there was obvious association between the high F-NLR score and poor DFS/ PFS/RFS (HR: 2.01; 95\% CI: 1.47-2.74). Sensitivity analysis indicated that the results of the meta-analysis were stable. Based on the above results, we have sufficient reasons to believe that F-NLR score may be a suitable and effective prognostic indicator for DSCs in clinical practice.

F-NLR score, based on fibrinogen levels and neutrophil and lymphocyte counts, was first identified as an effective prognostic indicator in ESCC (4). Subsequently, its prognostic value was also confirmed in numerous other tumors, such as glioblastoma multiforme, ovarian cancer, and non-small cell lung cancer (23-25). Similarly, increasing evidence suggests that the F-NLR score could be a good predictive marker in DSCs. Fibrinogen, as an acutephase response protein, has been associated with poor prognosis in patients with various tumors (26-28). NLR, as a useful marker for the assessment of inflammatory response, is calculated by dividing the neutrophil count by the lymphocyte count. A number of studies have reported that elevated NLR is associated with poor prognosis in patients with various malignancies, including DSCs (29-31). F-NLR score combined pretreatment fibrinogen level with neutrophil-lymphocyte ratio better reflects inflammatory responses and the cancer microenvironment. Fibrinogen or NLR alone may exert a limited effect on tumor progression. The F-NLR score overcomes the unfavorable effect of fibrinogen and NLR, and effectively improves the predicted value for patients with DSCs.

Several mechanisms may explain that F-NLR score can be used as an effective predictor in DSC. Fibrinogen is a key factor in hemostasis, which induces cell growth and migration, and is often abnormally activated in patients with cancer (32). When stimulated with inflammatory 
Table 2 Subgroup analysis of the studies reporting the effect of high F-NLR score in OS

\begin{tabular}{|c|c|c|c|c|c|c|}
\hline Stratified study & No. of studies & No. of patients & Pool HR $(95 \%$ Cl) & $P$ value & Heterogeneity $I^{2}(\%)$ & $P Q$ \\
\hline GC & 5 & 2,658 & 2.35 (1.89-2.91) & 0 & 0 & 0.808 \\
\hline EC & 5 & 1,237 & 1.82 (1.50-2.22) & 0 & 0 & 0.978 \\
\hline $\mathrm{HCC}$ & 2 & 422 & 2.12 (1.52-2.94) & 0 & 0 & 0.547 \\
\hline HPC & 1 & 111 & $1.99(0.90-4.38)$ & - & - & - \\
\hline Gl-tract cancers & 8 & 3,918 & 2.09 (1.78-2.45) & 0 & 42.90 & 0.092 \\
\hline Non-Gl-tract cancers & 8 & 1,770 & $1.90(1.61-2.25)$ & 0 & 0 & 0.985 \\
\hline \multicolumn{7}{|l|}{ Analysis type } \\
\hline With-surgery & 10 & 4,210 & $1.90(1.67-2.17)$ & 0 & 0 & 0.795 \\
\hline No-surgery & 3 & 277 & $2.13(1.47-3.09)$ & 0 & 0 & 0.959 \\
\hline Mixed & 3 & 1,201 & $2.92(1.61-5.29)$ & 0 & 67.5 & 0.046 \\
\hline \multicolumn{7}{|l|}{ Country } \\
\hline Japan & 6 & 1,456 & $2.22(1.71-2.88)$ & 0 & 0 & 0.837 \\
\hline China & 10 & 4,232 & $1.95(1.71-2.21)$ & 0 & 21.50 & 0.245 \\
\hline
\end{tabular}

GC, gastric cancer; EC, esophageal carcinoma; HCC, hepatocellular carcinoma; CRC, colorectal cancer; HPC, hypopharyngeal carcinoma; GI, gastrointestinal; OS, overall survival; F-NLR, fibrinogen and neutrophil-lymphocyte ratio.

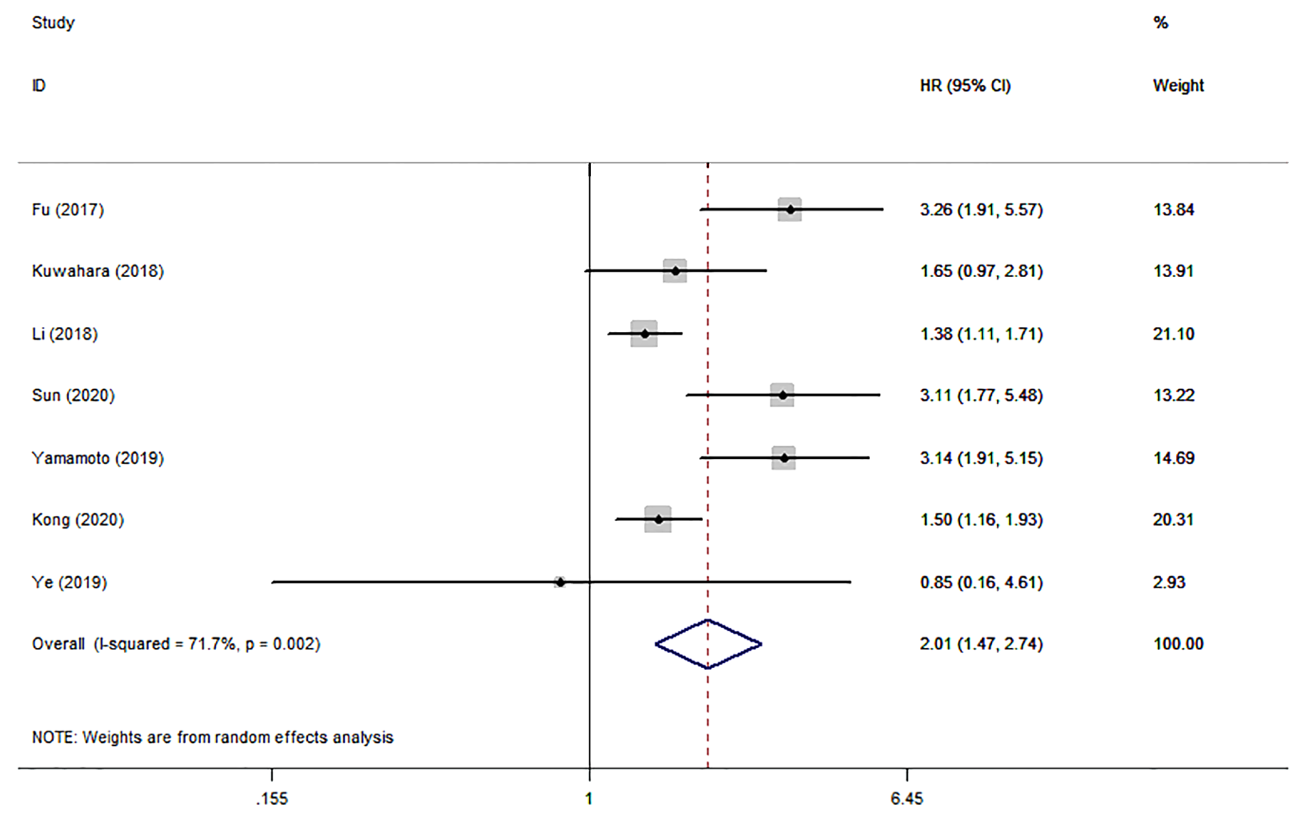

Figure 3 Forest plot of the relationship between pretreatment fibrinogen and neutrophil-lymphocyte ratio and disease-free survival/ progression-free survival/recurrence-free survival. 
Table 3 Analysis results based on DFS/PFS/RFS

\begin{tabular}{lcccccc}
\hline Stratified study & No. of studies & No. of patients & Pool HR $(95 \%$ Cl $)$ & P value & Heterogeneity I $^{2}(\%)$ & PQ \\
\hline DFS & 4 & 1,432 & $1.97(1.35-2.87)$ & 0 & 0.003 \\
RFS & 2 & 745 & $2.12(0.65-6.88)$ & 0.213 & 52.70 \\
PFS & 1 & 111 & $1.65(0.97-2.81)$ & - & - & - \\
\hline
\end{tabular}

DFS/PFS/RFS, disease-free survival/progression-free survival/recurrence-free survival.

A

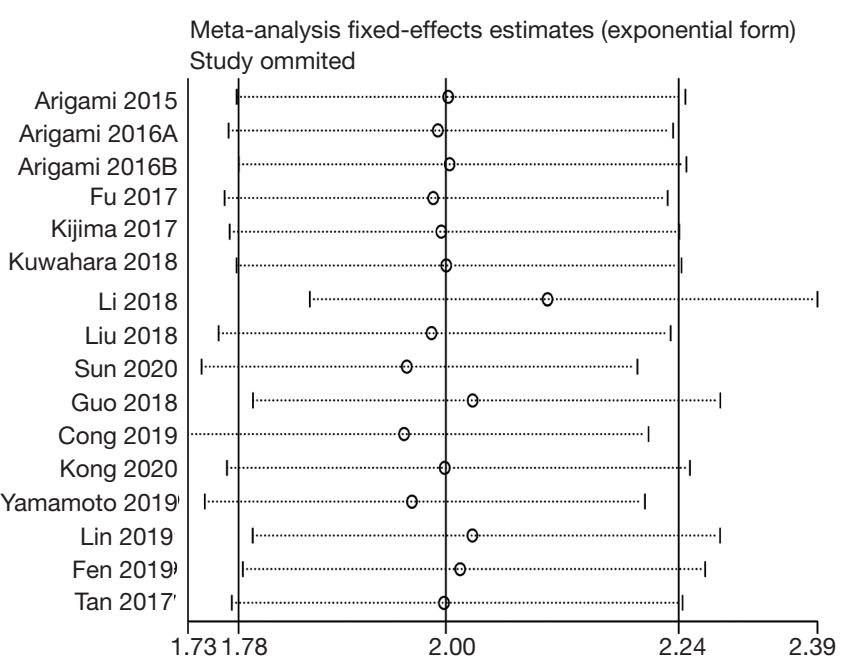

B

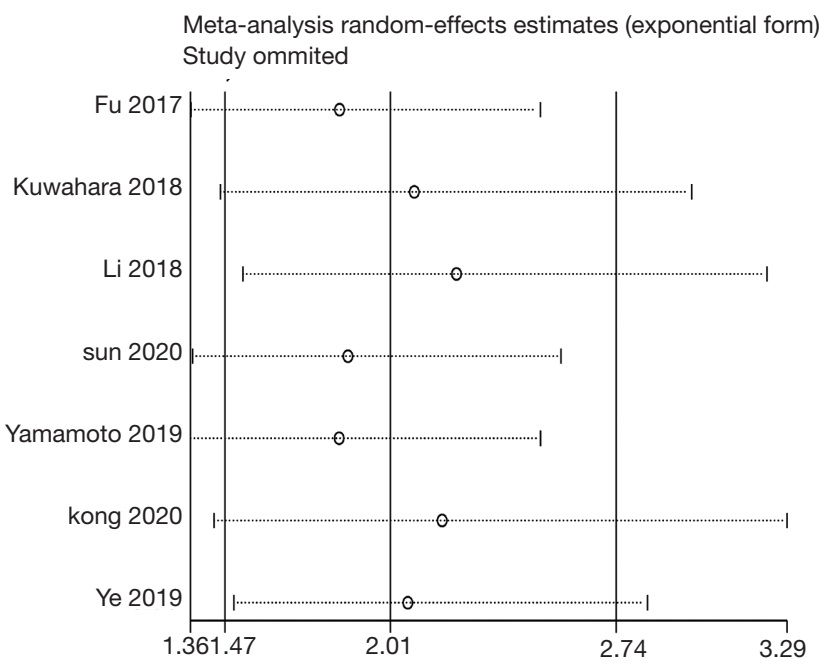

Figure 4 Funnel plot of sensitivity analysis. (A) Sensitivity analysis for overall survival. (B) Sensitivity analysis for disease-free survival/ progression-free survival/recurrence-free survival.
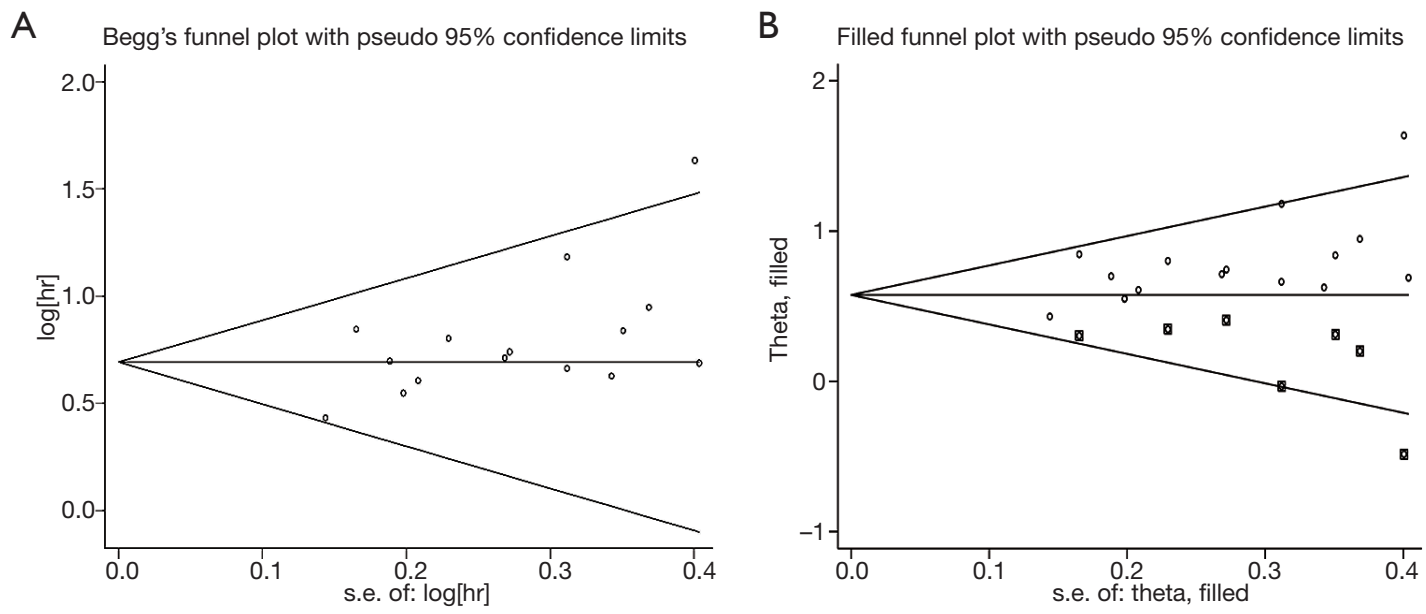

Figure 5 Funnel plots for publication bias for overall survival. (A) Begg's test to evaluate overall survival data. (B) Trim and fill to evaluate overall survival data. 


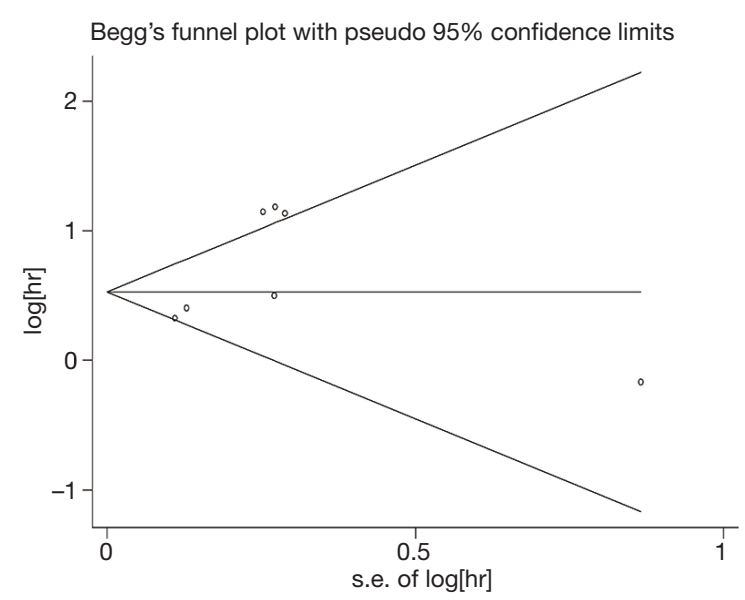

Figure 6 Funnel plots for publication bias for disease-free survival/ progression-free survival/recurrence-free survival.

factors or by tumors, activated thrombin can transform fibrinogen into fibrin, which can form a stable framework and extracellular matrix around tumor cells, preventing tumor cell killing by immune cells (33). It is established that tumor progression and prognosis is closely associated with inflammation $(34,35)$. Neutrophils, as a marker of inflammation, can promote tumor invasion, metastasis, and angiogenesis by producing various cytokines $(36,37)$. In addition, lymphocytes play important roles in anti-tumor immune defense, and their reduction may be considered an immune deficiency. Studies have reported that lymphopenia is associated with poor prognosis in GC (38). Therefore, high F-NLR, elevated fibrinogen, increased neutrophils, and decreased lymphocytes, represent intense inflammatory reactions and fragile immune response, which may contribute to the occurrence and development of tumors.

There were certain limitations in the present metaanalysis. Firstly, all included studies had small sample sizes, and their results may not be reliable. Secondly, some of the $\mathrm{HR}$ and CI values extracted from the survival curve may not be equal to the true value. Thirdly, all included studies were retrospective studies. Fourthly, most studies included in the meta-analysis were conducted in Asia. Future studies involving patients of different races and from various regions are warranted. Finally, publication bias existed in our analysis. This may be related to the different research methods and quality of included literature

Although there are some defects, this meta-analysis also has some strengths. Firstly, this was the first meta-analysis to investigate the relationship between the F-NLR score and prognostic outcomes in DSCs. Secondly, sensitivity analysis displayed that the results were stable. Thirdly, there was no heterogeneity for OS in the meta-analysis. Furthermore, the trim-and-fill method confirmed that the results of the meta-analysis were unaffected by the possible publication bias. More importantly, F-NLR score as the serum biomarker, is more convenient and rapid. This can be an efficacious method for dynamically monitoring the prognosis and therapeutic effects.

In summary, we demonstrated that the high F-NLR score is associated with poor prognostic outcomes in DSCs and may serve as an effective prognostic indicator in DSCs for the Asian population. Undoubtedly, further largesample, prospective, multicentric, and well-designed studies are warranted to validate the present results and explore the prognostic role of the F-NLR score in various types of cancer.

\section{Acknowledgments}

Funding: This work was supported by the Guangdong Natural Science Foundation (2016A030313278, 2015A030313038, 2015A030312013); Science and Technology Program of Guangzhou city (2014Y200200, 201604020001, 201508020262, 2014000000013, 201607010024); Science and Technology Program of Guangdong Province (2017B020209004, 20169013); National 13th Five-Year Science and Technology Plan Major Projects of China (2017ZX10203205-006-001); and Guangdong Key Laboratory of Liver Disease Research (2017B030314027).

\section{Footnote}

Reporting Checklist: The authors have completed the PRISMA reporting checklist. Available at http://dx.doi. org/10.21037/tcr-20-2482

Conflicts of Interest: All authors have completed the ICMJE uniform disclosure form (available at http://dx.doi. org/10.21037/tcr-20-2482). The authors have no conflicts of interest to declare.

Ethical Statement: The authors are accountable for all aspects of the work in ensuring that questions related to the accuracy or integrity of any part of the work are appropriately investigated and resolved.

Open Access Statement: This is an Open Access article 
distributed in accordance with the Creative Commons Attribution-NonCommercial-NoDerivs 4.0 International License (CC BY-NC-ND 4.0), which permits the noncommercial replication and distribution of the article with the strict proviso that no changes or edits are made and the original work is properly cited (including links to both the formal publication through the relevant DOI and the license). See: https://creativecommons.org/licenses/by-nc-nd/4.0/.

\section{References}

1. Siegel RL, Miller KD, Jemal A. Cancer statistics, 2016. CA Cancer J Clin 2016;66:7-30.

2. Jemal A, Center MM, DeSantis C, et al. Global patterns of cancer incidence and mortality rates and trends. Cancer Epidemiol Biomarkers Prev 2010;19:1893-907.

3. Chen W, Zheng R, Baade PD, et al. Cancer statistics in China, 2015. CA Cancer J Clin 2016;66:115-32.

4. Arigami T, Okumura H, Matsumoto M, et al. Analysis of the Fibrinogen and Neutrophil-Lymphocyte Ratio in Esophageal Squamous Cell Carcinoma: A Promising Blood Marker of Tumor Progression and Prognosis. Medicine (Baltimore) 2015;94:e1702.

5. Arigami $\mathrm{T}$, Uenosono $\mathrm{Y}$, Matsushita $\mathrm{D}$, et al. Combined fibrinogen concentration and neutrophil-lymphocyte ratio as a prognostic marker of gastric cancer. Oncol Lett 2016;11:1537-44.

6. Arigami T, Uenosono $\mathrm{Y}$, Ishigami S, et al. A Novel Scoring System Based on Fibrinogen and the NeutrophilLymphocyte Ratio as a Predictor of Chemotherapy Response and Prognosis in Patients with Advanced Gastric Cancer. Oncology 2016;90:186-92.

7. Fu SJ, Ji F, Han M, et al. Prognostic value of combined preoperative fibrinogen and neutrophil-lymphocyte ratio in patients with hepatocellular carcinoma after liver transplantation. Oncotarget 2017;8:4301-12.

8. Kijima T, Arigami T, Uchikado Y, et al. Combined fibrinogen and neutrophil-lymphocyte ratio as a prognostic marker of advanced esophageal squamous cell carcinoma. Cancer Sci 2017;108:193-9.

9. Kuwahara T, Takahashi H, Sano D, et al. Fibrinogen and Neutrophil-to-lymphocyte Ratio Predicts Survival in Patients with Advanced Hypopharyngeal Squamous Cell Carcinoma. Anticancer Res 2018;38:5321-30.

10. Li X, An B, Zhao Q, et al. Combined fibrinogen and neutrophil-lymphocyte ratio as a predictive factor in resectable colorectal adenocarcinoma. Cancer Manag Res 2018;10:6285-94.
11. Liu X, Liu Z, Lin E, et al. A cumulative score based on preoperative fibrinogen and the neutrophil-lymphocyte ratio to predict outcomes in resectable gastric cancer. Cancer Manag Res 2018;10:3007-14.

12. Sun $Y$, Zhang $Y$, Huang $Z$, et al. Combination of Preoperative Plasma Fibrinogen and Neutrophil-toLymphocyte Ratio (the F-NLR Score) as a Prognostic Marker of Locally Advanced Rectal Cancer Following Preoperative Chemoradiotherapy. World J Surg 2020;44:1975-84.

13. Tianxing G, Xiaojie P, Lihuan Z, et al. Combination of preoperative fibrinogen and neutrophil to lymphocyte ratio is a predictive prognostic factor in ESCC and AEG systematic review. Biosci Rep 2019;39:BSR20190480.

14. Cong X, Li S, Zhang Y, et al. The combination of preoperative fibrinogen and neutrophil-lymphocyte ratio is a predictive prognostic factor in esophagogastric junction and upper gastric cancer. J Cancer 2019;10:5518-26.

15. Kong W, Xu HH, Cheng JJ, et al. The Prognostic Role of a Combined Fibrinogen and Neutrophil-to-Lymphocyte Ratio Score in Patients with Resectable Hepatocellular Carcinoma: A Retrospective Study. Med Sci Monit 2020;26:e918824.

16. Yamamoto M, Kurokawa Y, Kobayashi N, et al. Prognostic Value of the Combined Index of Plasma Fibrinogen and the Neutrophil-Lymphocyte Ratio in Gastric Cancer. World J Surg 2020;44:207-12.

17. Lin JL, Guo TX, Pan XJ. Combined preoperative fibrinogen and neutrophil-lymphocyte ratio as a predictive factor in Esophageal Squamous Cell Carcinoma. Chin J Exp Surg 2019;36:2279-82.

18. Feng $Z$, Luo H, Sun YN, et al. Prognostic significance of combined fibrinogen concentration and neutrophil-tolymphocyte ratio in patients with Esophageal Squamous Cell Carcinoma receiving neoadjuvant therapy. Chin J Radiat Oncol 2019;28:188-92.

19. Qin L, Yao H, Xu L. Combined fibrinogen concentration and neutrophil-lymphocyte ratio as a prognostic marker of colorectal cancer. Chin J Immunology 2017;33:527-32.

20. Ye CM, Yi YD, Shen LB, et al. Combined fibrinogen concentration and neutrophil-lymphocyte ratio as a prognostic indicator for gastrointestinal stromal tumors. Chin J Gen Surg 2019;34:319-22.

21. Stang A. Critical evaluation of the Newcastle-Ottawa scale for the assessment of the quality of nonrandomized studies in meta-analyses. Eur J Epidemiol 2010;25:603-5.

22. Tierney JF, Stewart LA, Ghersi D, et al. Practical methods for incorporating summary time-to-event data into meta- 
analysis. Trials 2007;8:16.

23. Hao Y, Li XL, Chen HC, et al. A Cumulative Score Based on Preoperative Neutrophil-Lymphocyte Ratio and Fibrinogen in Predicting Overall Survival of Patients with Glioblastoma Multiforme. World Neurosurg 2019;128:e427-e433.

24. Marchetti C, Romito A, Musella A, et al. Combined Plasma Fibrinogen and Neutrophil Lymphocyte Ratio in Ovarian Cancer Prognosis May Play a Role? Int J Gynecol Cancer 2018;28:939-44.

25. Huang W, Wang SG, Zhang H, et al. Prognostic significance of combined fibrinogen concentration and neutrophil-to-lymphocyte ratio in patients with resectable non-small cell lung cancer. Cancer Biol Med 2018;15:88-96.

26. Cai HX, Li XQ, Wang SF. Prognostic value of fibrinogen and D-dimer-fibrinogen ratio in resectable gastrointestinal stromal tumors. World J Gastroenterol 2018;24:5046-56.

27. Sun ZQ, Han XN, Wang HJ, et al. Prognostic significance of preoperative fibrinogen in patients with colon cancer. World J Gastroenterol 2014;20:8583-91.

28. Xu H, Ai JZ, Tan P, et al. Pretreatment elevated fibrinogen level predicts worse oncologic outcomes in upper tract urothelial carcinoma. Asian J Androl 2020;22:177-83.

29. Sharaiha RZ, Halazun KJ, Mirza F, et al. Elevated preoperative neutrophil:lymphocyte ratio as a predictor of postoperative disease recurrence in esophageal cancer. Ann Surg Oncol 2011;18:3362-9.

Cite this article as: Liu R, Dai T, Zheng S, Deng M, Lin G, Bao Y, Guo Z, Wang G. Prognostic value of combined pretreatment fibrinogen and neutrophil-lymphocyte ratio in digestive system cancers: a meta-analysis of 17 retrospective studies. Transl Cancer Res 2021;10(1):241-250. doi: 10.21037/tcr20-2482
30. Absenger G, Szkandera J, Pichler M, et al. A derived neutrophil to lymphocyte ratio predicts clinical outcome in stage II and III colon cancer patients. Br J Cancer 2013;109:395-400.

31. Wang Z, Peng SH, Xie H, et al. Neutrophil-lymphocyte ratio is a predictor of prognosis in patients with castrationresistant prostate cancer: a meta-analysis. Cancer Manag Res 2018;10:3599-610.

32. Kwaan HC, Lindholm PF. Fibrin and Fibrinolysis in Cancer. Semin Thromb Hemost 2019;45:413-22.

33. Palumbo JS, Talmage KE, Massari JV, et al. Platelets and fibrin(ogen) increase metastatic potential by impeding natural killer cell-mediated elimination of tumor cells. Blood 2005;105:178-85.

34. Diakos CI, Charles KA, McMillan DC, et al. Cancerrelated inflammation and treatment effectiveness. Lancet Oncol 2014;15:e493-503

35. Coussens LM, Werb Z. Inflammation and cancer. Nature 2002;420:860-7.

36. Neagoe PE, Brkovic A, Hajjar F, et al. Expression and release of angiopoietin-1 from human neutrophils: intracellular mechanisms. Growth Factors 2009;27:335-44.

37. Gregory AD, Houghton AM. Tumor-associated neutrophils: new targets for cancer therapy. Cancer Res 2011;71:2411-6.

38. Wu ES, Oduyebo T, Cobb LP, et al. Lymphopenia and its association with survival in patients with locally advanced cervical cancer. Gynecol Oncol 2016;140:76-82. 